\title{
Ragweed-allergic subjects have decreased serum levels of chemokines CCL2, CCL3, CCL4 and CCL5 out of the pollen season
}

\author{
ZHIVKA KOSTOVA, TSVETELINA BATSALOVA, DZHEMAL MOTEN, IVANKA TENEVA, \\ BALIK DZHAMBAZOV
}

Plovdiv University, Plovdiv, Bulgaria

\begin{abstract}
$C C$-chemokines are important mediators of the allergic responses and regulate the cell trafficking. The aim of this study was to examine the serum levels of CCL2/MCP-1,CCL3/MIP-1 $\alpha, C C L 4 / M I P-1 \beta$ and CCL5/RANTES, and to determine whether there are differences between ragweed-allergic subjects and healthy individuals out of the pollen season. Peripheral blood samples were collected from 24 subjects allergic to ragweed pollen and 12 healthy controls. Serum concentrations of chemokines/ cytokines were measured by an enzyme-linked immunosorbent assay. We observed significantly decreased concentrations of CCL2/MCP-1,CCL3/MIP-1 $\alpha, C C L 4 / M I P-1 \beta$ and CCL5/RANTES in the sera of ragweed-allergic patients compared to the healthy individuals $(32.2 \mathrm{vs} .106 .4 \mathrm{pg} / \mathrm{ml}, 89.5 \mathrm{vs}$. $135.7 \mathrm{pg} / \mathrm{ml}, 63.4 \mathrm{vs} .119 .2 \mathrm{pg} / \mathrm{ml}$ and $11.2 \mathrm{vs} .18 .1 \mathrm{ng} / \mathrm{ml}$, respectively, $p<0.01)$. In contrast to the $C C$-chemokines, the serum levels of IL-8/CXCL8 showed a significant increase $(p<0.05)$ in the allergic group compared to the non-allergic subjects. Interleukin 4 levels were similar in both groups. In the sera of allergic patients, we have also detected significantly elevated levels of ragweed-specific IgE and IgG. However, decreased serum concentrations of the four CC-chemokines and elevated levels of IL-8/ CXCL8 can be used as biomarkers for more accurate evaluation of the allergic status of patients with pollen allergy out of the season, to study the mechanisms for activation/inhibition of the subclinical allergic responses and for development of therapeutic strategies.
\end{abstract}

Key words: chemokines, IgE, pollen allergy, Ambrosia artemisiifolia, ragweed.

(Cent Eur J Immunol 2015; 40 (4): 442-446)

\section{Introduction}

Chemokines are small chemoattractant cytokines that regulate cell trafficking and play a major role in various immune responses including allergic reactions. Based on the genomic organization of the human and mouse chemokine genes and the number and location of the cysteine residues at the $\mathrm{N}$-terminus, these molecules have been classified into four major groups, named CXC, CC, XC and CX3C [1]. CCL2 (monocyte chemoattractant protein-1, MCP-1), CCL3 (macrophage inflammatory protein-1 $\alpha$, MIP-1 $\alpha$, LD78 $\alpha$ ), CCL4 (macrophage inflammatory protein-1 $\beta$, MIP-1 $\beta$ ) and CCL5 (regulated on activation, normal $\mathrm{T}$ cell expressed and secreted, RANTES) belong to the CC subfamily and their genes in humans are localized on chromosome 17 [1]. These four chemokines are chemotactic for monocytes, T lymphocytes, basophils and NK cells, although they function through separate receptors. MCP-1 and RANTES are potent histamine-releasing factors for basophils. In addition, RANTES can attract or activate eosinophils [2].

Several studies demonstrated the role of CC chemokines in airway allergic inflammation. Increased levels of MCP-1, MIP- $1 \alpha$, MIP-1 $\beta$ or RANTES have been detected in the bronchoalveolar lavage fluid (BALF) of patients with active asthma [3, 4], with mustard gas-induced pulmonary fibrosis $[5,6]$, or in the nasal secretions of individuals after allergen challenge [7] or during the pollen season [8]. Ghazanfari et al. also reported significantly elevated levels of MCP-1 in the sera of sulfur mustard exposed individuals compared to control subjects, but in contrast to the other reports, they found significantly decreased levels of RANTES suggesting a different pathophysiology and diverse molecular mechanisms involved in this type of intoxication [9]. Greatly enhanced BALF levels of MCP-1, MIP- $1 \alpha$ and RANTES were detected $4 \mathrm{~h}$ after the endobronchial allergen challenge of stable asthmatic patients compared to the saline-challenged control subjects. The 
concentration of these three chemokines returned to the baseline levels $24 \mathrm{~h}$ after the challenge [10].

Radman and colleagues showed increased serum levels of MCP-1 during the active symptoms (anaphylactic reaction or asthma attack) in children with food allergies, but they were not able to detect RANTES in the tested samples [11]. Interesting results have been reported by Glez et al. [12] whereas they observed significantly lower levels of MCP-1 and MIP- $1 \alpha$ in the sera of children with persistent allergy to cow's milk protein compared to children with atopy who do not have this allergy. Therefore, still little is known about the role of the serum levels of these chemokines for activation or inhibition of certain allergic reactions. The objective of the present study was to investigate whether there are any differences between serum basal concentrations of CCL2/MCP-1, CCL3/MIP-1 $\alpha$, CCL4/MIP-1 $\beta$ and CCL5/RANTES in healthy and ragweed-allergic subjects out of the pollen season.

\section{Material and methods}

\section{Study design}

Twenty-four patients (fifteen males and nine females, 25-40 years old, mean age 31 ) with ragweed pollen allergy and 12 healthy non-allergic age-matched subjects (nine males and three females, 23-40 years old, mean age 29) were recruited for this study during the period of September-November 2013 in collaboration with an allergologist. Inclusion criteria for the allergic patients were based on the case history (ragweed pollen induced seasonal allergic rhinitis for at least two years), positive skin prick test to ragweed pollen (ALK-Abelló A/S Hřrsholm, Denmark) and serum specific IgE to ragweed pollen extract (measured by ImmunoCAP, ImmunoDiagnostics Thermo Fisher Scientific, Uppsala, Sweden). Non-allergic controls were identified as having negative skin prick tests to the main groups of allergens and no clinical history of allergy. Furthermore, none of the subjects had received allergen-specific immunotherapy or used antihistamines or corticosteroids during the past 2 months before the blood sampling. All participants were non-smokers.

This study was conducted outside the pollen season (in February 2014) and none of the patients showed allergic symptoms. All tests and analyses were performed in adherence to the Declaration of Helsinki ethical guidelines. The local Ethics Committee has approved the study, and informed consent was obtained from all participants.

\section{Serum collection}

Peripheral venous blood samples were collected out of the ragweed pollen season (February) into Venoject tubes for serum sampling and centrifuged for 15 minutes at $1500 \times \mathrm{g}$ at room temperature. The serum was transferred immediately to $2-\mathrm{ml}$ microtubes and stored at $-70^{\circ} \mathrm{C}$ until analyses.

\section{Measurement of chemokine/cytokine levels in the serum}

Serum levels of the chemokines/cytokines were determined using commercially available Human Common Chemokines/Cytokines Multi-Analyte ELISArray Kits (QIAGEN) according to the manufacturer's instructions. Study samples and standard dilutions of the chemokines/ cytokines were assayed in duplicate. The absorbance was read at $450 \mathrm{~nm}$ with the correction set to $570 \mathrm{~nm}$ by using a microplate reader (BioTek, USA). The limits of detection for the assay was $10 \mathrm{pg} / \mathrm{ml}$ for each chemokine/ cytokine.

\section{Measurement of ragweed pollen specific $\mathbf{I g E}$ and IgG}

Levels of IgE and IgG specific to ragweed pollen were measured by ELISA. Microtiter plates (MaxiSorp Immuno Plate, Nalge Nunc International, Roskilde, Denmark) were coated with $50 \mu \mathrm{l} /$ well pollen extract from Ambrosia artemisiifolia L. (1000 PNA/ml, BB-NCIPD Ltd., Sofia, Bulgaria) overnight at $4^{\circ} \mathrm{C}$. After blocking for $1 \mathrm{~h}$ with phosphate-buffered saline (PBS), $0.05 \%$ (v/v) Tween 20 and $1 \%(\mathrm{w} / \mathrm{v})$ non-fat milk, the plates were washed with PBS/Tween 20 and $1: 10$ diluted sera from the allergic and non-allergic (healthy) subjects were applied onto the coated plates $(100 \mu \mathrm{l} /$ well $)$ in triplicates and incubated overnight at $4{ }^{\circ} \mathrm{C}$. After washing, $50 \mu \mathrm{l}$ of biotinylated monoclonal anti-human IgE antibody (1 : 1000 dilution, clone GE-1, Sigma-Aldrich) or biotinylated anti-human IgG ( $\gamma$-chain specific) antibody ( $1: 2000$ dilution, Sigma-Aldrich) was added for $1 \mathrm{~h}$ at $37^{\circ} \mathrm{C}$ for detection of ragweed pollen specific $\operatorname{IgE}$ and $\mathrm{IgG}$, respectively. Antibodies were detected using peroxidase-conjugated goat anti-biotin pAb (Calbiochem ${ }^{\circledR}$, Merck Millipore) and 2,2-azino-di-[2-ethylbenzthiazoline sulfonate] diammonium salt (ABTS tablets, Roche Diagnostics) as substrate. The absorbance values were determined by a microplate reader (BioTek, USA) at $405 \mathrm{~nm}$. All OD values were counted as positive if they exceeded the mean OD of the negative controls by more than three standard deviations.

\section{Statistical analysis}

Data were analysed using the StatView software (SAS Institute, USA). Since the probability distribution of the chemokines/cytokines levels within the investigated groups (allergic and healthy subjects) are not known in advance, we have used non-parametric tests. The Kolmogorov-Smirnov test was used to analyse the distribution, and Mann-Whitney $U$ test to determine the levels of difference between the two groups. All data are presented as means and standard deviations. Values of $p<0.05$ were considered statistically significant. 


\section{Results}

To identify whether there are any differences in the signalling molecules between patients with pollen allergy and non-allergic individuals out of the pollen season, we have collected blood samples from all participants within 5 days in February 2014. Data from all 36 individuals (24 patients with ragweed pollen allergy and 12 healthy controls) were included in the analyses. Figure 1 shows the levels of CCL2/MCP-1, CCL3/MIP-1 $\alpha$, CCL4/MIP-1 $\beta$,

A

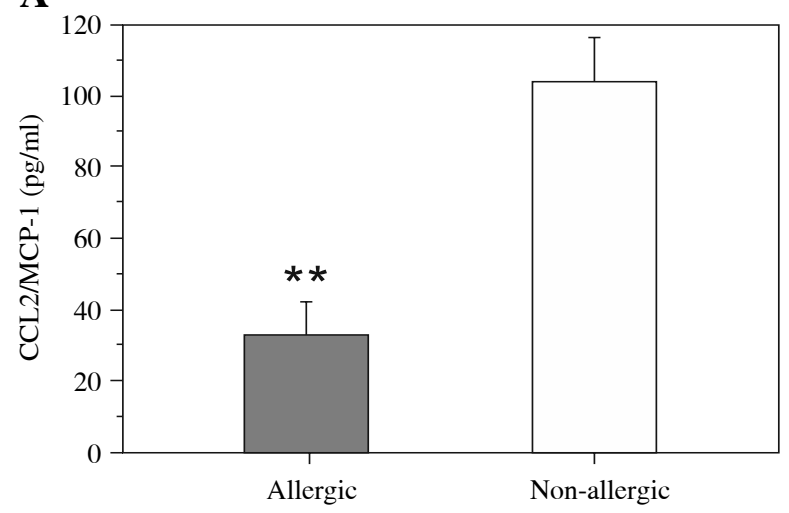

C

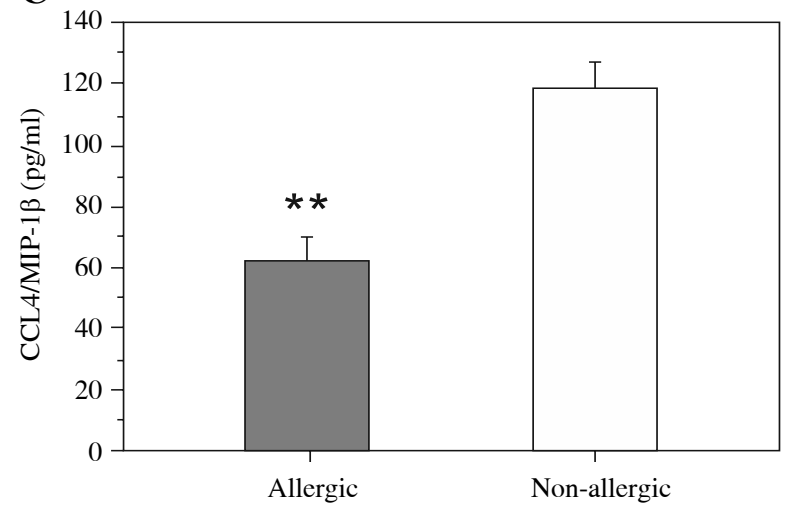

E

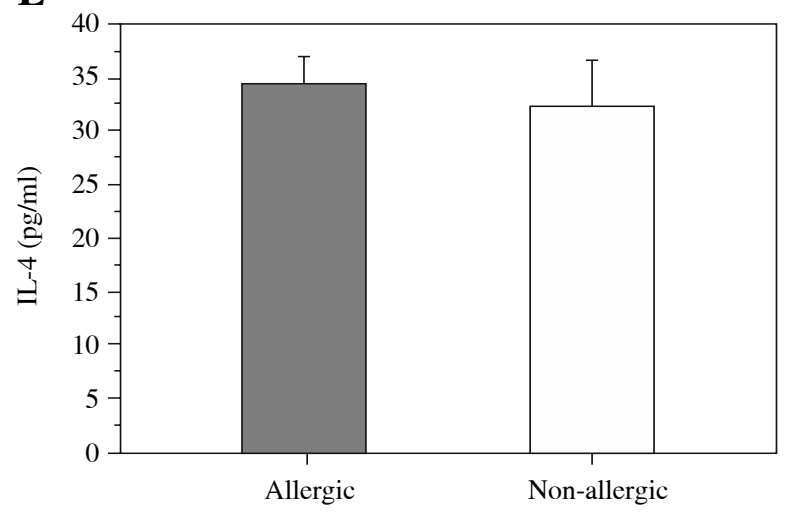

CCL5/RANTES, IL-4 and IL-8/CXCL8 in the sera of ragweed-allergic and control (non-allergic) subjects. As can be seen in Fig. 1A, the serum level of CCL2/MCP1 in the allergic patients $(32.2 \pm 4.7 \mathrm{pg} / \mathrm{ml}, n=14)$ was approximately 3.5 -fold less than controls $(106.4 \pm 5.8 \mathrm{pg} /$ $\mathrm{ml}, n=12, p<0.01)$. Results presented in Fig. 1B-D indicate that the serum levels of CCL3/MIP-1 $\alpha$, CCL4/ MIP- $1 \beta$ and CCL5/RANTES are also decreased significantly in the pollen-sensitive subjects compared to

B

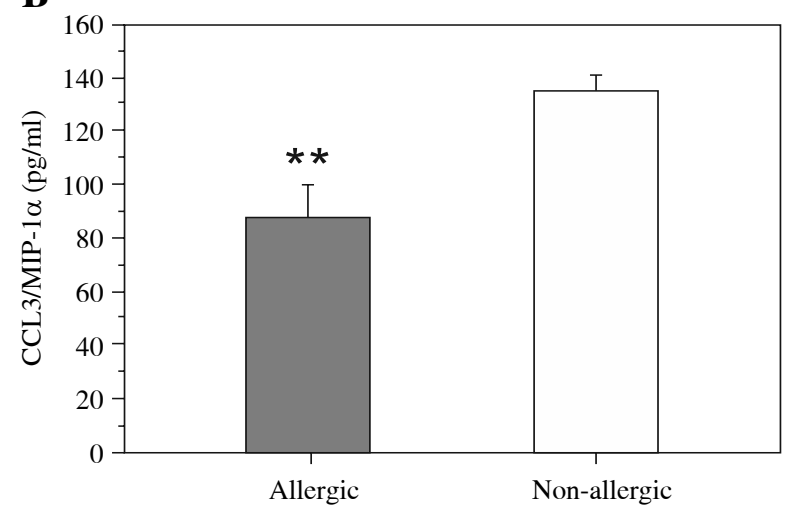

D

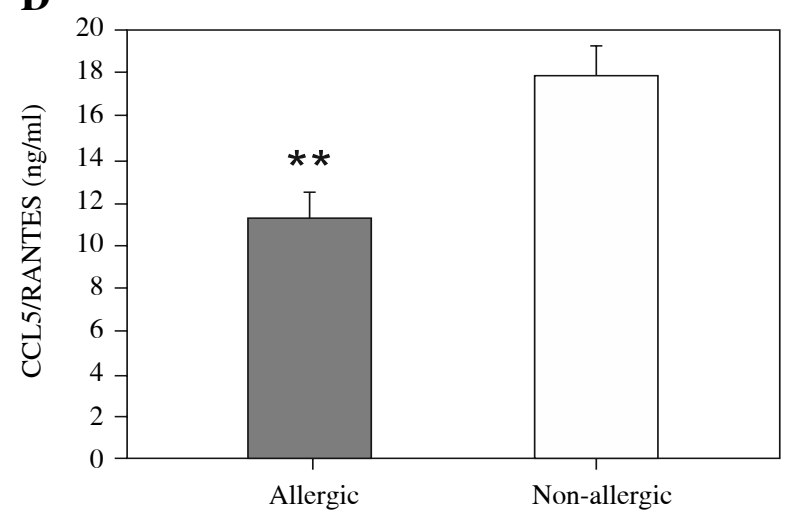

F

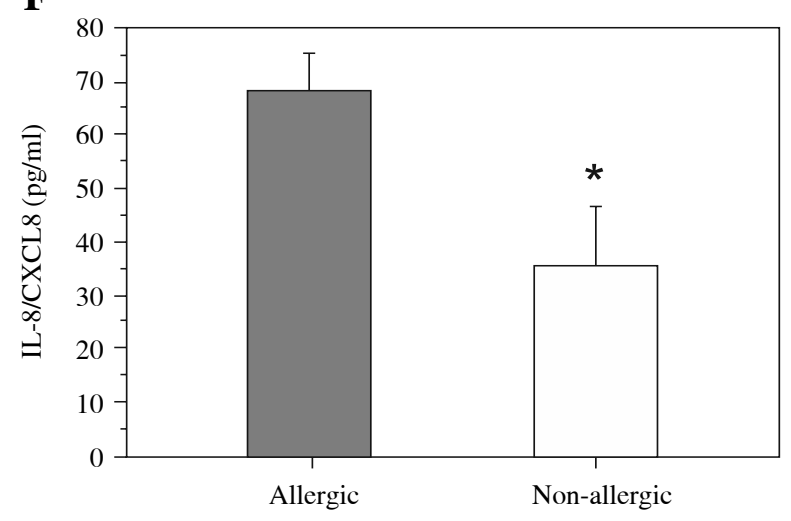

$* p<0.05, * * p<0.01$.

Fig. 1. Serum concentrations of CCL2/MCP-1 (A), CCL3/MIP-1 $\alpha$ (B), CCL4/MIP-1 $\beta$ (C), CCL5/RANTES (D), IL-4 $(\mathbf{E})$ and IL-8/CXCL8 $(\mathbf{F})$ in patients with ragweed pollen allergy $(n=24)$ and non-allergic individuals $(n=12)$. Blood samples were collected out of the pollen season. The results are presented as mean \pm SD 
A

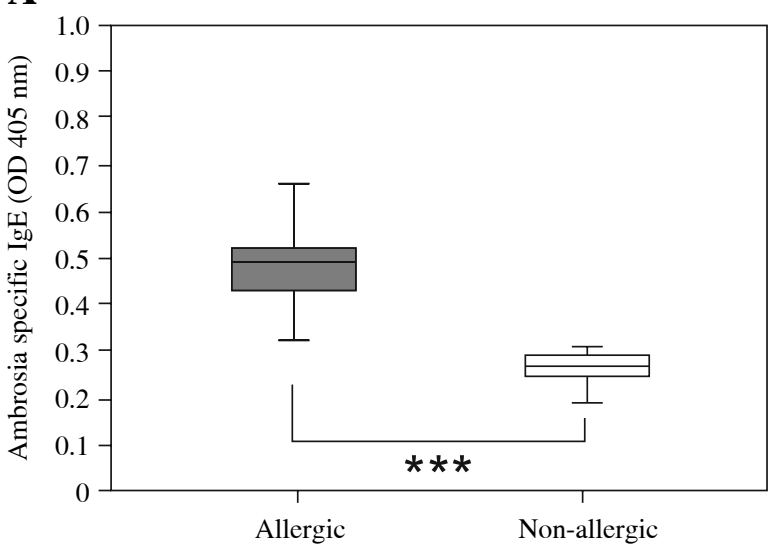

B

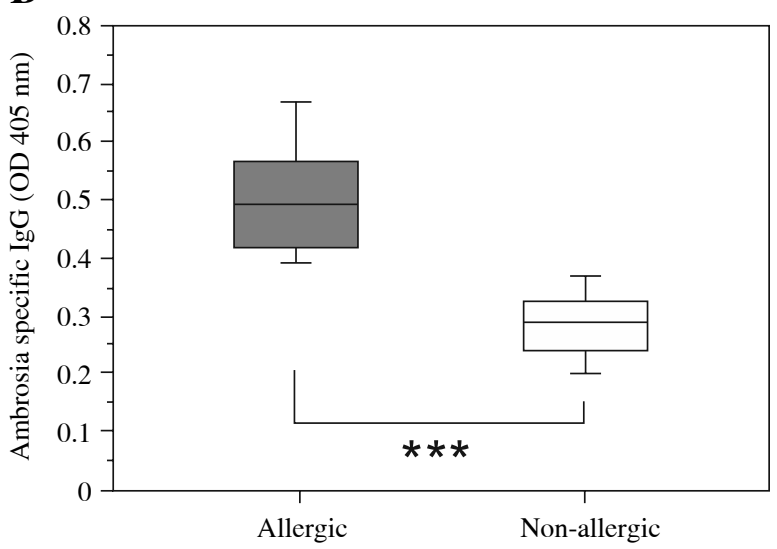

$* * * p<0.001$

Fig. 2. Serum levels of specific $\operatorname{IgE}(\mathbf{A})$ and $\operatorname{IgG}(\mathbf{B})$ antibodies to pollen extract from ragweed (Ambrosia artemisiifolia $\mathrm{L}$.) in patients with ragweed pollen allergy $(n=24)$ and non-allergic subjects $(n=12)$ out of the pollen season. The results are presented as mean $\pm \mathrm{SD}$

the control group (89.5 vs. $135.7 \mathrm{pg} / \mathrm{ml}, 63.4$ vs. 119.2 $\mathrm{pg} / \mathrm{ml}$ and $11.2 \mathrm{vs} .18 .1 \mathrm{ng} / \mathrm{ml}$, respectively, $p<0.01)$. However, our data showed that out of the pollen season the allergic patients have significantly lower serum levels of the four chemokines in comparison with the healthy individuals.

We next assessed the serum levels of IL-4 and IL-8 (CXCL8) in the same allergic and healthy individuals. As indicated in Fig. 1E, out of the pollen season, there was no statistical difference in circulating levels of IL-4 between healthy and allergic subjects. Interleukin 4 concentrations were similar in both groups $(32-35 \mathrm{pg} / \mathrm{ml})$. In contrast, the serum levels of IL-8 showed a significant increase $(p<0.05)$ in the allergic group compared to the non-allergic control group (Fig. 1F).

Serum samples were additionally analysed for presence of ragweed pollen-specific IgE and IgG. We detected significantly higher levels of $\operatorname{IgE}$ in the sera of allergic patients compared to healthy controls $(p<0.001)$ (Fig. 2A). Similarly, ragweed-specific IgG levels were also significantly higher in patients with pollen allergy than in non-allergic individuals $(p<0.001)$ (Fig. 2B). However, out of the pollen season, allergic patients still have high levels of free pollen-specific antibodies although the clinical symptoms of the allergy are not present.

\section{Discussion}

The serum levels of different chemokines in subjects with pollen allergy during the pollen season and out of the season are poorly investigated. Our hypothesis was that any differences in the chemokine levels between allergic and non-allergic individuals out of the pollen season may contribute to the activation/inhibition and pathophysiology of this allergic disorder. We evaluated the serum levels of four more relevant CC-chemokines such as CCL2/MCP-1, CCL3/MIP-1 $\alpha$, CCL4/MIP-1 $\beta$ and CCL5/RANTES. These chemokines are known as potent chemotactic factors for basophils, eosinophils and mononuclear cells [3, 1315]. They are also involved in the activation of eosinophils and basophils during the allergic inflammation leading to basophil secretion of histamine $[8,16]$.

Our study was focused on the serum levels of the chemokines since it was conducted out of the pollen season when the allergic patients do not have any clinical symptoms. We have shown that out of the pollen season the allergic patients have significantly lower serum levels of investigated four CC-chemokines compared to healthy controls. Decreased serum levels of the four CC-chemokines in the allergic patients suggest that the threshold level for activation of their receptors in allergic subjects is lower compared to non-allergic individuals or there is an increased expression of the chemokine receptors in allergic patients. Thus, keeping a low concentration of the CC-chemokines is a mechanism to protect initiation of an allergic inflammatory reaction by these chemokines at a physiologic concentration.

Investigated four CC-chemokines act mainly via the receptors CCR2 and CCR5 [15]. Our data support the hypothesis of Zhang et al. [17] that the CCR5+CCR2+ expressing cells are long-term memory cells that can respond rapidly to submaximal stimulation with any inflammatory chemokines. Therefore, the lower concentration of the chemokines in the allergic subjects out of the season prevents from rapid responses of these memory cells. Our data about the chemokines can be used for diagnostic purposes and for development of an effective protocol for treatment 
of pollen-allergic individuals similarly to the desensitization method applied by Glez et al. [12].

Decreased levels of IL-8 (CXCL8) in the nasal secretions of atopic subjects with symptoms during the ragweed season as well as in the sera of sulfur mustard-exposed individuals were reported by Kuna et al. [8] and Ghazanfari et al. [9], respectively. Out of the pollen season, we found elevated serum levels of IL-8, which is consistent with the reported data taking in account that IL-8 inhibits release of histamine. This is in agreement and supports our suggestion for presence of an additional mechanism that protects the organism from a rapid allergic response.

We found no significant differences in the serum levels of IL-4 between allergic and non-allergic individuals out of the pollen season, which is consistent with the observation of Gabrielsson et al. [18] that IL-4 and IL-13 producing cells exist only during the pollen season in the circulation of type I allergic patients and not in healthy individuals. During the pollen season the same authors also detected increased levels of allergen-specific IgE. Our analyses showed increased levels of ragweed pollen-specific antibodies (IgE and $\mathrm{IgG}$ ) several months after the pollen season. Similarly to our results, Carlsoon et al. [19] reported that highest IgE levels may be found 3-4 months after the pollen season. The explanation is that production of antibodies is initiated during the pollen season, and after expansion of B cells and their maturation to plasma cells, the highest levels of antibodies occur several months after the initial trigger [19]. These experiments showed that the presence or absence of IgE cannot be used by itself as a clinical biomarker for accurate determination of the allergic status.

In conclusion, we showed significantly decreased serum levels of CCL2/MCP-1, CCL3/MIP-1 $\alpha$, CCL4/ MIP-1 $\beta$ and CCL5/RANTES and elevated levels of IL-8/ CXCL8 in allergic patients out of the pollen season compared to healthy controls. These chemokines can be used to study the mechanism for activation/inhibition of the subclinical response outside the season in patients with pollen allergy as well as for more accurate evaluation of the allergic status and development of the therapeutic strategy for pollen desensitization. BF-002

This work was financially supported by grant MU-11/

The authors declare no conflicts of interest.

\section{References}

1. Zlotnik A, Yoshie O (2012): The chemokine superfamily revisited. Immunity 36: 705-716.

2. Rollins BJ (1997): Chemokines. Blood 90: 909-928.

3. Alam R, York J, Boyars M, et al. (1996): Increased MCP-1, RANTES, and MIP-1alpha in bronchoalveolar lavage fluid of allergic asthmatic patients. Am J Respir Crit Care Med 153: 1398-1404.

4. Miotto D, Christodoulopoulos P, Olivenstein R, et al. (2001): Expression of IFN-gamma-inducible protein; monocyte chemotactic proteins 1,3 , and 4 ; and eotaxin in TH1- and TH2-mediated lung diseases. J Allergy Clin Immunol 107: 664-670.

5. Emad A, Emad V (2007): Elevated levels of MCP-1, MIP-alpha and MIP-1 beta in the bronchoalveolar lavage (BAL) fluid of patients with mustard gas-induced pulmonary fibrosis. Toxicology 240: 60-69.

6. Emad A, Emad Y (2007): Relationship between eosinophilia and levels of chemokines (CCL5 and CCL11) and IL-5 in bronchoalveolar lavage fluid of patients with mustard gas-induced pulmonary fibrosis. J Clin Immunol 27: 605-612.

7. Sim TC, Reece LM, Hilsmeier KA, et al. (1995): Secretion of chemokines and other cytokines in allergen-induced nasal responses: inhibition by topical steroid treatment. Am J Respir Crit Care Med 152: 927-933.

8. Kuna P, Lazarovich M, Kaplan AP (1996): Chemokines in seasonal allergic rhinitis. J Allergy Clin Immunol 97: 104112.

9. Ghazanfari T, Yaraee R, Kariminia A, et al. (2009): Alterations in the serum levels of chemokines 20 years after sulfur mustard exposure: Sardasht-Iran Cohort Study. Int Immunopharmacol 9: 1471-1476.

10. Holgate ST, Bodey KS, Janezic A, et al. (1997): Release of RANTES, MIP-1 alpha, and MCP-1 into asthmatic airways following endobronchial allergen challenge. Am J Respir Crit Care Med 156: 1377-1383.

11. Radman M, Hassanshahi G, Vazirinejad R, et al. (2013): Serum levels of the CC chemokines CCL2, CCL5, and CCL11 in food allergic children with different clinical manifestations. Inflammation 36: 561-566.

12. Glez PP, Franco YB, Matheu V (2012): MIP-1alpha, MCP-1, and desensitization in anaphylaxis from cow's milk. N Engl J Med 367: 282-284.

13. Alam R, Stafford S, Forsythe P, et al. (1993): RANTES is a chemotactic and activating factor for human eosinophils. J Immunol 150: 3442-3448.

14. Schall TJ, Bacon K, Camp RD, et al. (1993): Human macrophage inflammatory protein alpha (MIP-1 alpha) and MIP-1 beta chemokines attract distinct populations of lymphocytes. J Exp Med 177: 1821-1826.

15. Borish LC, Steinke JW (2003): 2. Cytokines and chemokines. J Allergy Clin Immunol 111: S460-S475.

16. Kuna P, Reddigari SR, Schall TJ, et al. (1993): Characterization of the human basophil response to cytokines, growth factors, and histamine releasing factors of the intercrine/ chemokine family. J Immunol 150: 1932-1943.

17. Zhang HH, Song K, Rabin RL, et al. (2010): CCR2 identifies a stable population of human effector memory CD4+ $\mathrm{T}$ cells equipped for rapid recall response. J Immunol 185: 6646-6663.

18. Gabrielsson S, Soderlund A, Paulie S, et al. (1998): Increased frequencies of allergen-induced interleukin-13-producing cells in atopic individuals during the pollen season. Scand J Immunol 48: 429-435.

19. Carlsson M, Thorell L, Sjolander A, Larsson-Faria S (2015): Variability of total and free IgE levels and IgE receptor expression in allergic subjects in and out of pollen season. Scand J Immunol 81: 240-248. 